\title{
ERRATA TO VOLUME 109
}

P. M. Cohn. Noncommutative unique factorization domains, pp. 313-331.

Page 317 , line 10. The characterization of 'prime' should read: $a$ is prime if and only if $R / a R$ has no submodules other than 0 or itself with a strictly cyclic quotient. (For definition of 'strictly cyclic' see p. 326.)

Page 317. Proof of Proposition 2.2. After ' $R$ to $a R$ ' insert: with strictly cyclic quotients.

Page 319. The proofs of Theorems 3.2 and 3.3 are valid only if $R$ is also a weak Bezout ring. This does not affect later sections (which are only concerned with weak Bezout rings). 